\title{
Effective genome editing and identification of a regiospecific gallic acid 4-O-glycosyltransferase in pomegranate (Punica granatum L.)
}

\author{
Lijing Chang ${ }^{1,2}$, Sheng $\mathrm{Wu}^{1,2}$ and Li Tian $\mathbb{1}^{1,2,3}$
}

\begin{abstract}
Pomegranate (Punica granatum L.) trees are woody perennials that bear colorful and nutritious fruits rich in phenolic metabolites, e.g., hydrolyzable tannins (HTs) and flavonoids. We here report genome editing and gene discovery in pomegranate hairy roots using Clustered Regularly Interspaced Short Palindromic Repeats (CRISPR)/CRISPR-associated protein 9 (Cas9) (CRISPR/Cas9), coupled with transcriptome and biochemical analyses. Single guide RNAs (sgRNAs) were designed to target two UDP-dependent glycosyltransferases (UGTs), PgUGT84A23 and PgUGT84A24, which possess overlapping activities in $\beta$-glucogallin (a galloylglucose ester; biosynthetic precursor of HTs) biosynthesis. A unique accumulation of gallic acid 3-O- and 4-O-glucosides (galloylglucose ethers) was observed in the PgUGT84A23 and PgUGT84A24 dual CRISPR/Cas9-edited lines (i.e., ugt84a23 ugt84a24) but not the control (empty vector) or PgUGT84A23/PgUGT84A24 single edited lines (ugt84a23 or ugt84a24). Transcriptome and real-time qPCR analyses identified 11 UGTs with increased expression in the ugt84a23 ugt84a24 hairy roots compared to the controls. Of the 11 candidate UGTs, only PgUGT72BD1 used gallic acid as substrate and produced a regiospecific product gallic acid 4-O-glucoside. This work demonstrates that the CRISPR/Cas9 method can facilitate functional genomics studies in pomegranate and shows promise for capitalizing on the metabolic potential of pomegranate for germplasm improvement.
\end{abstract}

\section{Introduction}

The woody plant pomegranate (Punica granatum L.) produces colorful flowers and fruits with ornamental and culinary values. Different pomegranate tissues have historically been used for alleviating symptoms or treating various diseases due to the accumulation of a wide diversity of bioactive metabolites ${ }^{1}$. In recent years, pomegranate fruits and juice have been pursued by consumers for their favorable nutritional quality, contributed by the abundant phenolic compounds, e.g., hydrolyzable tannins (HTs) and flavonoids, in these tissues and

Correspondence: Li Tian (Itian@ucdavis.edu)

${ }^{1}$ Shanghai Key Laboratory of Plant Functional Genomics and Resources, Shanghai Chenshan Botanical Garden, 201602 Shanghai, China

${ }^{2}$ Shanghai Chenshan Plant Science Research Center, Chinese Academy of Sciences, 201602 Shanghai, China

Full list of author information is available at the end of the article. products $^{2}$. Genetic variations underlying different metabolite profiles reportedly exist in pomegranate and have been utilized for breeding cultivars with desirable traits ${ }^{3}$. Complementary to the classic breeding approach, new molecular techniques, such as genome editing, can enable targeted modification of key metabolic genes for improved nutritional and commercial quality of pomegranate fruits and products.

Among the various genome-editing technologies, Clustered Regularly Interspaced Short Palindromic Repeats (CRISPR)/CRISPR-associated protein 9 (Cas9) (CRISPR/Cas9) has gained increasing popularity for its efficiency and ease of use. In this method, a single guide RNA (sgRNA) directs the Cas9 nuclease to the target gene sequence upstream of a protospacer adjacent motif. Cas9 creates a break in the double-strand DNA, which is then ligated by homology-directed repair or non-homologous

\section{(c) The Author(s) 2019}

(c) (i) Open Access This article is licensed under a Creative Commons Attribution 4.0 International License, which permits use, sharing, adaptation, distribution and reproduction cc) in any medium or format, as long as you give appropriate credit to the original author(s) and the source, provide a link to the Creative Commons license, and indicate if changes were made. The images or other third party material in this article are included in the article's Creative Commons license, unless indicated otherwise in a credit line to the material. If material is not included in the article's Creative Commons license and your intended use is not permitted by statutory regulation or exceeds the permitted use, you will need to obtain permission directly from the copyright holder. To view a copy of this license, visit http://creativecommons.org/licenses/by/4.0/. 


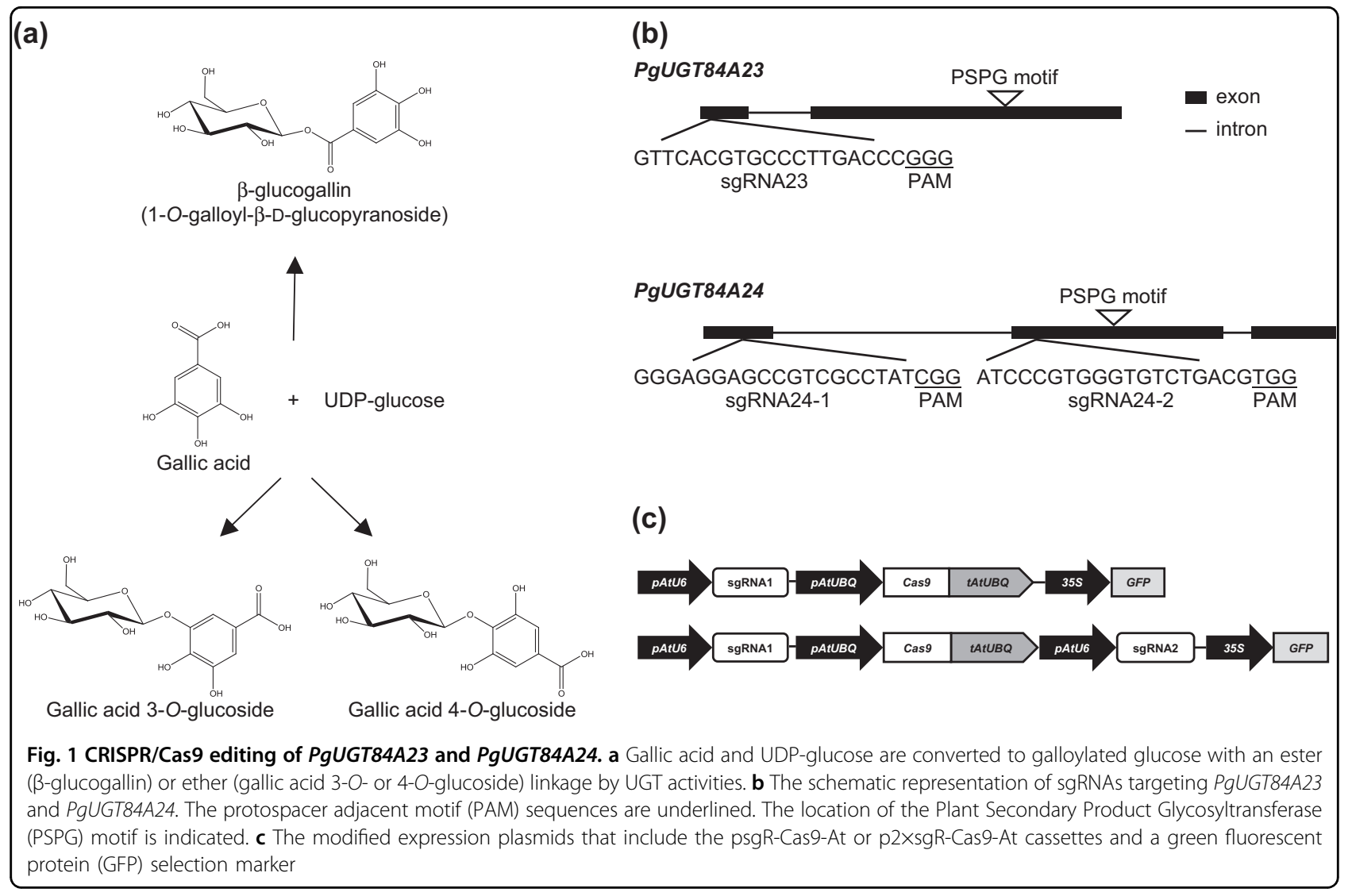

end joining ${ }^{4}$. In general, five genotypes can be obtained from the CRISPR/Cas9-mediated genome editing in a diploid species, including wild type (no mutations), homozygous mutant (same mutations in both alleles), heterozygous mutant/monoallelic (mutation in one allele, wild type in the other allele), biallelic (different mutations in the two alleles), and chimera (more than two different mutations in the alleles $)^{5}$. Initially used for disruption of gene function, there has been rapid advancement in the CRISPR/Cas9 technology for more precise (e.g., base editing) and versatile (e.g., controlling gene expression) genome editing ${ }^{6,7}$.

Although CRISPR/Cas9 has been successfully adopted in many plant species (e.g., Arabidopsis, tobacco, tomato, rice etc.), its application has not been reported in pomegranate $^{8}$. In consideration of the time and effort required for transformation and regeneration of pomegranate plants, we chose a hairy root system for testing the feasibility and efficacy of CRISPR/Cas9-mediated genome editing in pomegranate. This is because hairy roots can be induced from different pomegranate explants, accumulate HTs and other phenolic compounds, are transformable, and produce sufficient amounts of tissues for molecular and metabolite analyses within 3 months of transformation?
To select an easily discernable phenotype for verification of successful genome editing in pomegranate hairy roots, we chose PgUGT84A23 and PgUGT84A24, encoding two UDP-dependent glycosyltransferases (UGTs) that form $\beta$-glucogallin from gallic acid and UDP-glucose, as target genes (Fig. 1a) ${ }^{10}$. Reduced accumulation of punicalagin $\alpha$ and $\beta$ isomers (the most abundant HTs in pomegranate; produced from $\beta$-glucogallin) was observed in pomegranate hairy roots with attenuated $P g$ UGT84A23 and PgUGT84A24 activities (via RNAi suppression of PgUGT84A23 and PgUGT84A24 gene expression $)^{10}$. Therefore, the punicalagin levels in hairy roots can serve as a metabolic phenotype for knocking out PgUGT84A23 and PgUGT84A24 activities through genome editing.

In this work, we generated pomegranate hairy root lines containing CRISPR/Cas9-edited PgUGT84A23 and/or PgUGT84A24. We also modified the expression plasmids by incorporating a green fluorescent protein (GFP) marker for rapid and non-destructive screening of transgenic hairy roots. Metabolite analysis was conducted on the control (empty vector) as well as the single and dual CRISPR/Cas9-edited hairy roots (i.e., ugt84a23, ugt84a24, and ugt84a23 ugt84a24) and showed significant changes in ugt84a23 ugt84a24. Comparative transcriptome analysis was subsequently carried out on the control and 
ugt84a23 ugt84a24 hairy roots, which led to the identification of a new regioselective UGT toward gallic acid.

\section{Results}

The CRISPR/Cas9-sgRNAs effectively created mutations in PgUGT84A23 and PgUGT84A24

To knockout the activity of PgUGT84A23 or PgUGT84A24, one sgRNA for PgUGT84A23 (sgRNA23) and two sgRNAs for PgUGT84A24 (sgRNA24-1 and sgRNA24-2) were designed, which are specific for each target gene and away from the Plant Secondary Product Glycosyltransferase (PSPG) motif conserved among plant UGTs for binding sugar donors (Fig. 1b). To eliminate both PgUGT84A23 and PgUGT84A24 activities,
sgRNA23 and sgRNA24-1/sgRNA24-2 were placed into the same expression plasmid (Fig. 1c). A GFP selection marker was incorporated in the plasmid for sgRNA and Cas9 expression and used for screening of transgenic hairy roots (Fig. 1c).

Two hundred pomegranate hairy roots were transformed with each sgRNA or sgRNA combinations (i.e., sgRNA23, sgRNA24-1, sgRNA24-2, sgRNA23+sgRNA24-1, or sgRNA23+sgRNA24-2) and about $80 \%$ of the transformants exhibited green fluorescence emission upon excitation. Multiple GFP-positive hairy roots derived from each expression plasmid were randomly selected for sequencing (Tables 1 and 2). Of the seven sgRNA23 hairy root lines analyzed, there were two homozygous mutants

Table 1 Mutations identified in the pomegranate ugt84a23 or ugt84a24 hairy root lines

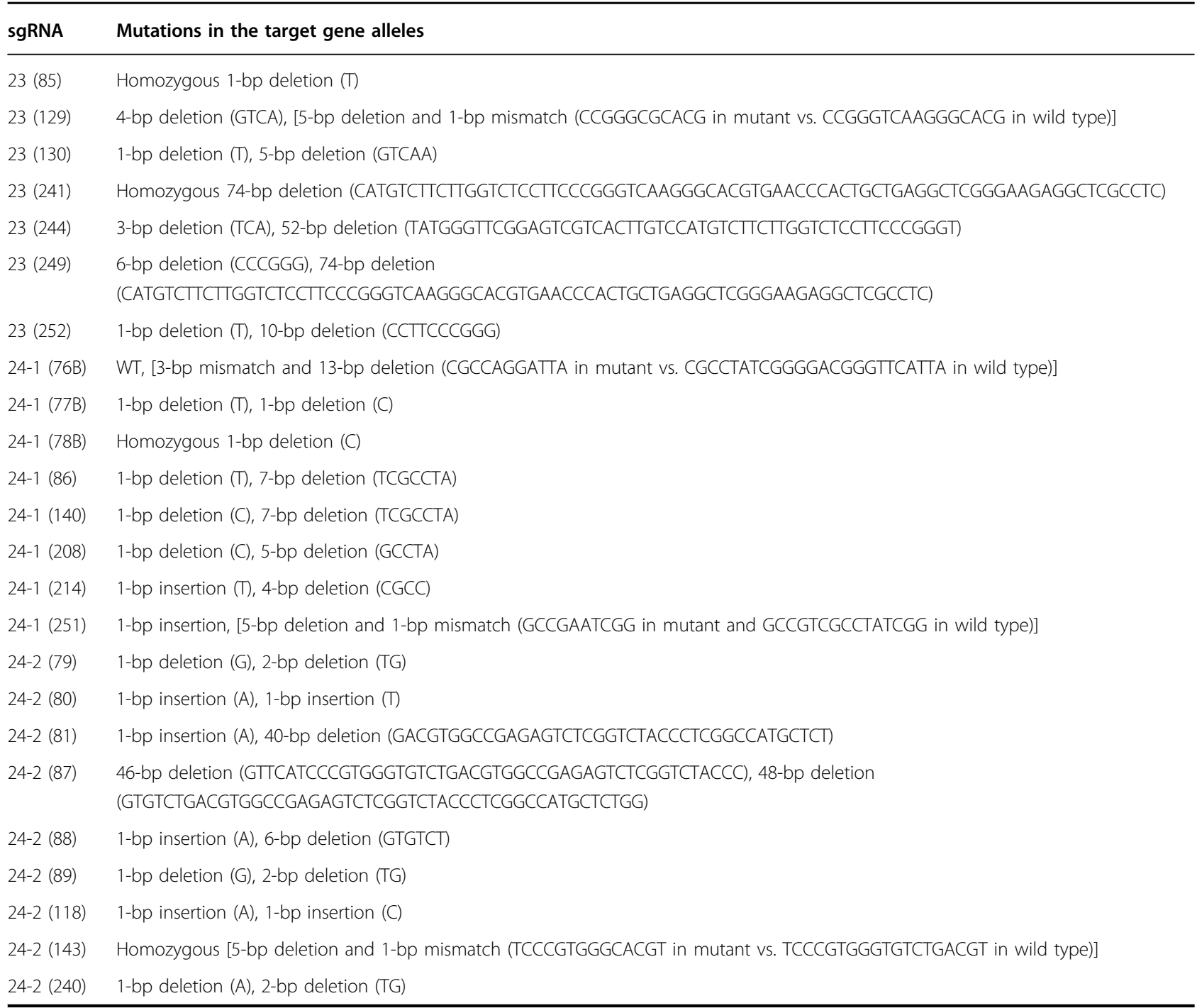

sgRNA23 (23) targets PgUGT84A23, whereas sgRNA24-1 (24-1) and sgRNA24-2 (24-2) target different regions of PgUGT84A24. Specific hairy root line numbers are shown in parenthesis next to the sgRNA designation. For mutations that include a deletion and a mismatch, both the mutant and the reference wild-type sequences are shown

WT, wild-type allele 
Table 2 Mutations identified in the pomegranate ugt84a23 ugt84a24 hairy root lines

\begin{tabular}{|c|c|c|}
\hline \multirow[t]{2}{*}{ sgRNA } & \multicolumn{2}{|c|}{ Mutations in the target gene alleles } \\
\hline & PgUGT84A23 & PgUGT84A24 \\
\hline $23+24-1(305)$ & $\begin{array}{l}\text { WT, 6-bp deletion (GGCACG), } \\
\text { 7-bp deletion } \\
\text { (CCGGGTC), 13-bp insertion } \\
\text { (TGAATTAGTTAGT) }\end{array}$ & 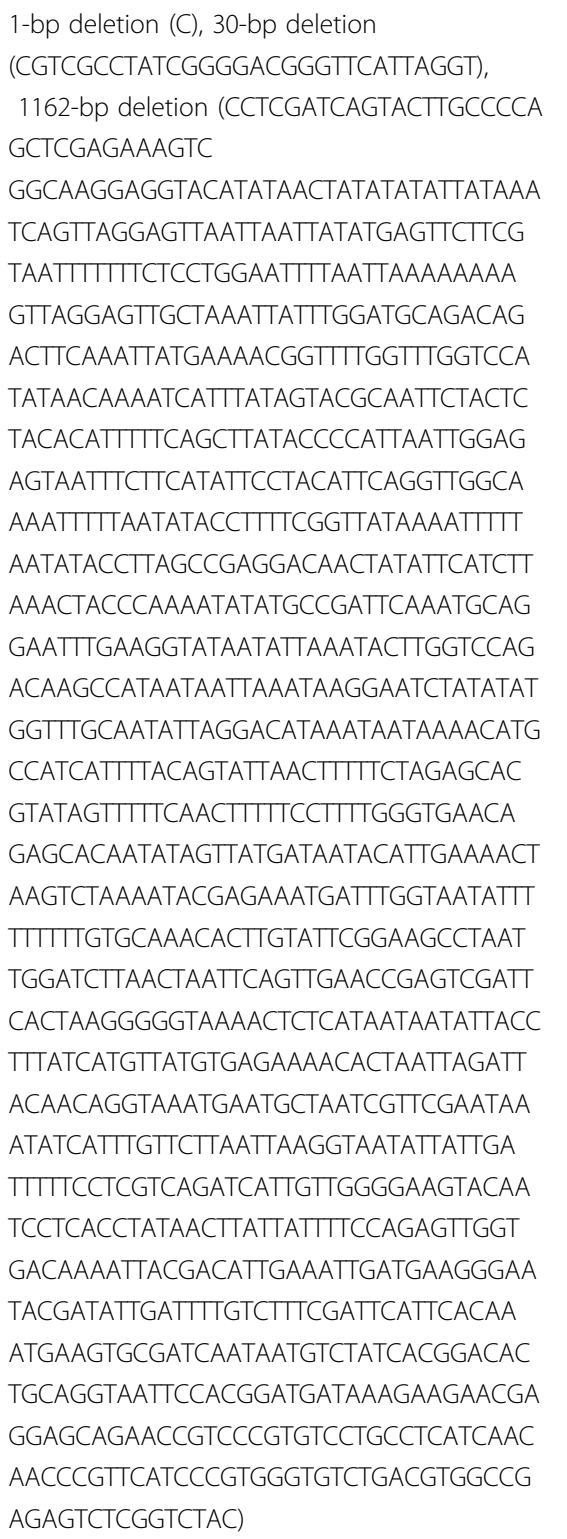 \\
\hline $23+24-1(307)$ & $\begin{array}{l}\text { Homozygous } \\
\text { 2-bp deletion (TC) }\end{array}$ & $\begin{array}{l}\text { 3-bp deletion (GCC), 19-bp deletion (CGGGGAGG } \\
\text { AGCCGTCGCCT) }\end{array}$ \\
\hline $23+24-1(310)$ & $\begin{array}{l}\text { Homozygous 10-bp deletion } \\
\text { (GTCAAGGGCA) }\end{array}$ & Homozygous 1-bp deletion (C) \\
\hline $23+24-1(316)$ & Homozygous 1-bp deletion (G) & 1-bp deletion (C), 3-bp deletion (CGC) \\
\hline $23+24-1(318)$ & $\begin{array}{l}\text { 1-bp mismatch (G/T), 3-bp } \\
\text { deletion (TCA), 9-bp deletion } \\
\text { (GTCAAGGGC), 20-bp deletion } \\
\text { (CTTGGTCTCCTTCCCGGGTC), }\end{array}$ & WT, 18-bp deletion (GAGCCGTCGCCTATCGGG) \\
\hline
\end{tabular}


Table 2 continued

\begin{tabular}{|c|c|c|}
\hline \multirow[t]{2}{*}{ sgRNA } & \multicolumn{2}{|l|}{ Mutations in the target gene alleles } \\
\hline & PgUGT84A23 & PgUGT84A24 \\
\hline & $\begin{array}{l}\text { 25-bp deletion (TTCTTGGTCTC } \\
\text { CTTCCCGGGTCAAG) }\end{array}$ & \\
\hline $23+24-1(319)$ & Homozygous 1-bp deletion (T) & 1-bp deletion (T), 29-bp deletion (CGTCGCCTATCGGGGACGGGTTCATTAGG) \\
\hline $23+24-1(321)$ & $\begin{array}{l}\text { WT, [21-bp mismatch } \\
\text { and 6-bp deletion } \\
\text { (GAAGGGTTCACCCGACCACAC in mutant vs. } \\
\text { TTCCCGGGTCAAGGGCACGTGAACCCA in wild type)] }\end{array}$ & 1-bp deletion (T), 8-bp deletion (CGTCGCCT) \\
\hline $23+24-1(322)$ & Homozygous 2-bp insertion (GC) & 6-bp deletion (GTCGCC), 7-bp insertion (TCCTTTT) \\
\hline $23+24-1(323)$ & Homozygous 1-bp deletion (G) & $\begin{array}{l}\text { 3-bp deletion (CGC), 21-bp deletion (GAGGAGCCGTCG } \\
\text { CCTATCGGG) }\end{array}$ \\
\hline $23+24-1(324)$ & 1-bp deletion (G), 3-bp deletion (GGG) & 1-bp deletion (T), 6-bp deletion (TCGCCT) \\
\hline $23+24-1(325)$ & 3-bp deletion (TCA), 4-bp deletion (TCAA) & 6-bp deletion (GCCGTC), 7-bp deletion (TCGCCTA) \\
\hline $23+24-1(327)$ & 1-bp insertion (T), 5-bp deletion (GGGTC) & 1-bp insertion (A), 3-bp deletion (CGC) \\
\hline $23+24-1(346)$ & 4-bp deletion (TCAA), 7-bp deletion (CCGGGTC) & $\begin{array}{l}\text { 21-bp deletion (GAGGAGCCGTCGCCTATCGGG), 68-bp deletion } \\
\text { (CGCCTATCGGGGACGGGTTCATTAGGTTCGAGTTCTITGAAGATG } \\
\text { GATGGGACGAGGATGAGCCCCGG) }\end{array}$ \\
\hline $23+24-2(309)$ & 1-bp mismatch (A/T), 9-bp deletion (TCAAGGGCA) & $\begin{array}{l}\text { 2-bp deletion (TG), 59-bp deletion (CAACAACCCGTTCATCC } \\
\text { CGTGGGTGTCTGACGTGGCCGAGAGTCTCGGTCTACCCTCGG) }\end{array}$ \\
\hline $23+24-2(312)$ & $\begin{array}{l}\text { 1-bp mismatch (C/T), 12-bp deletion } \\
\text { (CGGGTCAAGGGC) }\end{array}$ & WT, 1-bp insertion (T) \\
\hline $23+24-2(313)$ & $\begin{array}{l}\text { WT, 1-bp deletion (G), 5-bp deletion (GTCAA), } \\
\text { 124-bp deletion (TCTCGAATTATTAGCCGC } \\
\text { AGAAGAAGAAGCAGAAGAAGGAATTACAGG } \\
\text { TGAATTAGTTAGTTCATTATGGGTTCGGAGTCGT } \\
\text { CACTTGTCCATGTCTTCTTGGTCTCCTTCCCGGGTCAAGGGC) }\end{array}$ & 5-bp deletion (GTCTG), 7-bp deletion (GTCTGAC) \\
\hline $23+24-2(328)$ & 1-bp deletion (G), 3-bp deletion (TCA) & 1-bp insertion (T), 6-bp deletion (TGACGT) \\
\hline $23+24-2(333)$ & $\begin{array}{l}\text { WT, 3-bp deletion (GTC), } \\
\text { 10-bp deletion (GGGTCAAGGG) }\end{array}$ & $W T$, 1-bp insertion (A), 1-bp insertion (T) \\
\hline $23+24-2(335)$ & $\begin{array}{l}\text { 3-bp deletion (GTC), } \\
\text { 35-bp deletion (TCACTTGTCCATGTC } \\
\text { TTCTTGGTCTCCTTCCCGGG) }\end{array}$ & 1-bp insertion (A), 1-bp deletion (G) \\
\hline
\end{tabular}

sgRNA23 (23) targets PgUGT84A23, whereas sgRNA24-1 (24-1) and sgRNA24-2 (24-2) target different regions of PgUGT84A24. Specific hairy root line numbers are shown in parenthesis next to the sgRNA designation. For mutations that include a deletion and a mismatch, both the mutant and the reference wild-type sequences are shown. The ugt84a23 ugt84a24 mutants that were subjected to transcriptome sequencing are highlighted in bold

WT, wild-type allele

with a 1-bp deletion or a 74-bp deletion and five biallelic mutants carrying deletions (or a deletion and a mismatch) of different sizes (Table 1). For sgRNA24-1, one homozygous mutant of a 1-bp deletion, one heterozygous mutant with a 3-bp mismatch and a 13-bp deletion in one allele, and six biallelic mutants were detected (Table 1). For sgRNA24-2, one homozygous mutant of a combined 5-bp deletion and 1-bp mismatch and eight biallelic mutants with deletions and/or insertions were identified (Table 1).
For the 36 dual sgRNA lines (sgRNA23+sgRNA24-1/ sgRNA24-2) selected for sequencing, 23 were identified as homozygous, biallelic, or chimeric mutants for PgUGT84A24 and further examined for mutations in the PgUGT84A23 alleles, 19 of which also showed homozygous, biallelic, heterozygous, or chimeric mutations in PgUGT84A23 (Table 2). Interesting variations of CRISPR/ Cas9 editing were observed in the dual sgRNA lines, e.g., a long deletion of 1162-bp in a PgUGT84A24 allele in sgRNA23+sgRNA24-1 (line 305) and both a 21-bp 


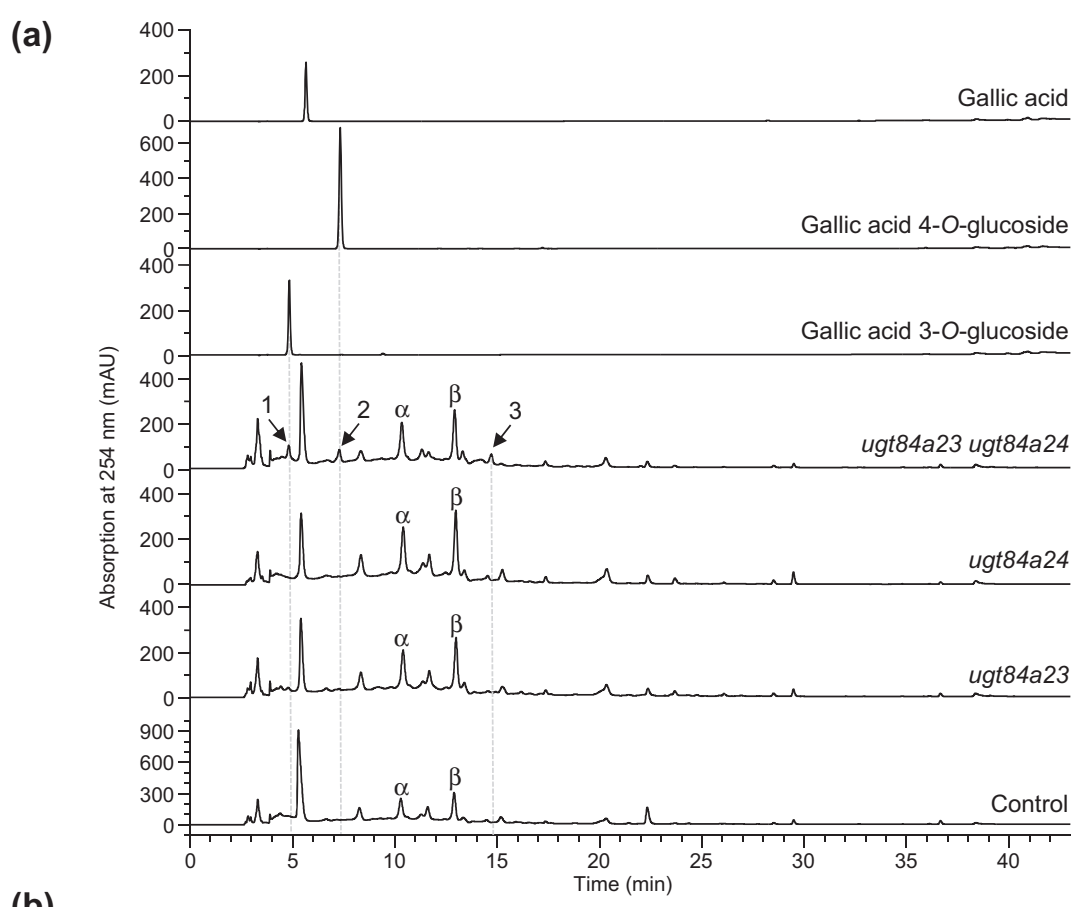

(b)
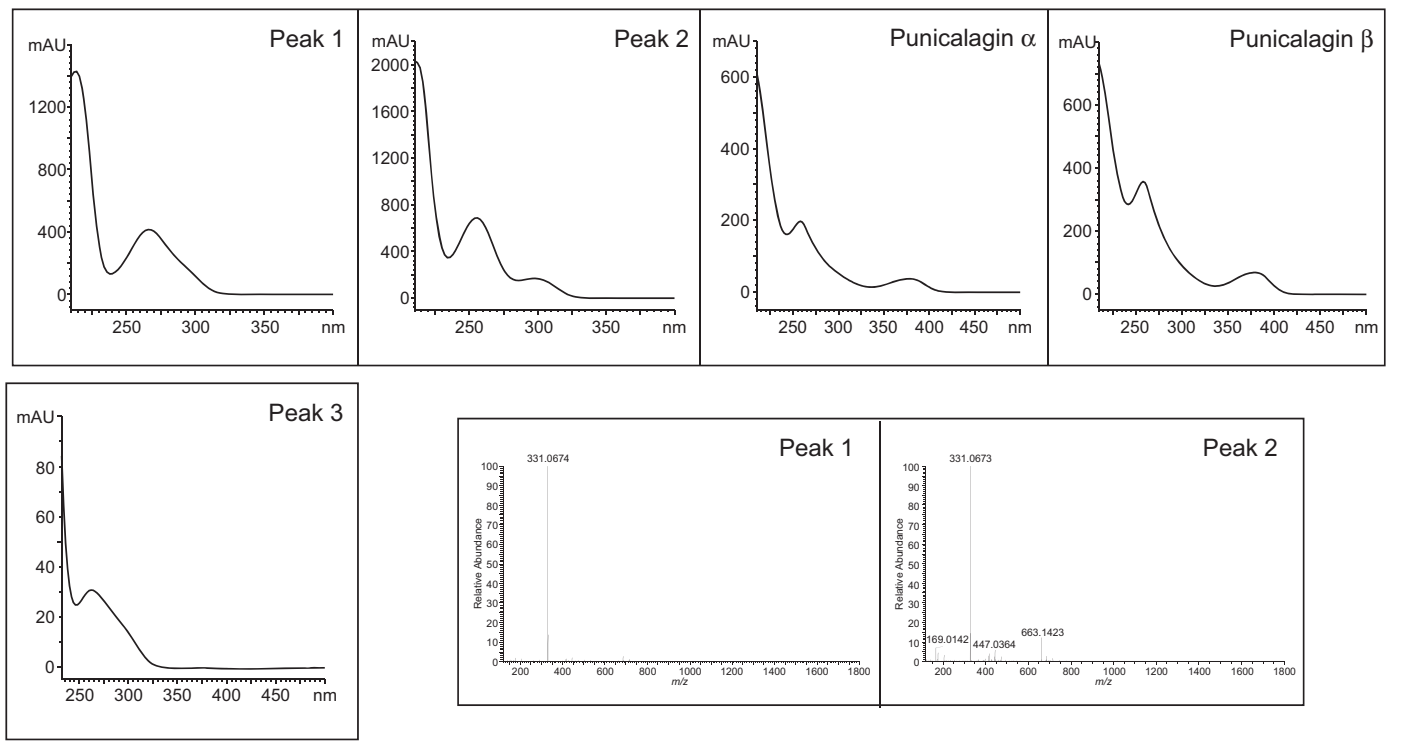

(c)

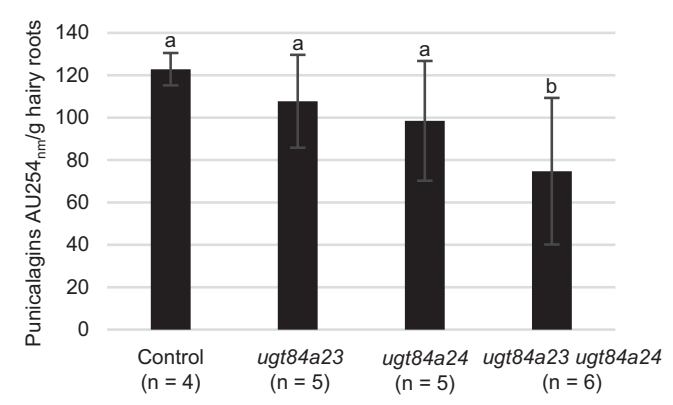

Fig. 2 (See legend on next page.) 
(see figure on previous page)

Fig. 2 Metabolite analysis of the control and the CRISPR/Cas9-edited PgUGT84A23 and/or PgUGT84A24 hairy roots. a HPLC chromatograms of authentic standards as well as pomegranate hairy roots containing the vector plasmid (control) or the CRISPR/Cas9-edited PgUGT84A23 and/or PgUGT84A24 (ugt84a23, ugt84a24, and ugt84a23 ugt84a24). Representative chromatograms of the hairy root lines are shown. Peaks (1-3) are only present in ugt84a23 ugt84a24 hairy roots and indicated with arrows. a, punicalagin $\mathbf{a} ; \beta$, punicalagin $\beta$. $\mathbf{b}$ Absorption spectra of peaks 1-3 and punicalagin $a$ and $\beta$ isomers. Mass spectra of peaks eluted at 4.85 min (peak 1) and $7.32 \mathrm{~min}$ (peak 2) are shown. $\mathbf{c}$ Peak areas of punicalagins (punicalagin $\alpha$ and $\beta$ isomers) in the control and the CRISPR/Cas9-edited PgUGT84A23 and/or PgUGT84A24 hairy root lines. Different letters indicate significant differences $(P<0.05)$ in punicalagin peak areas

Table 3 UGTs that showed significantly increased expression in the ugt84a23 ugt84a24 hairy roots compared to the control hairy roots in the transcriptome analysis

\begin{tabular}{llclc}
\hline Gene name & Control (TPM) & ugt84a23 ugt84a24 (TPM) & Log $_{2}$ FC & Adjusted $P$ value \\
\hline Pgr010311 & 0.81 & 5.24 & 2.34 & $6.16 \mathrm{E}-08$ \\
Pgr000395 & 1.55 & 7.46 & 2.11 & $3.00 \mathrm{E}-05$ \\
Pgr008782 & 4.07 & 19.06 & 2.02 & $1.04 \mathrm{E}-07$ \\
Pgr025855 & 3.39 & 16.01 & 2.01 & $3.37 \mathrm{E}-07$ \\
Pgr026914 & 5.75 & 27.64 & 1.79 & $5.10 \mathrm{E}-04$ \\
PgUGT72BD1 & 8.66 & 32.65 & 1.60 & $2.75 \mathrm{E}-03$ \\
Pgr023854 & 0 & 0.45 & 1.60 & $3.00 \mathrm{E}-02$ \\
Pgr011620 & 0.22 & 2.27 & 1.60 & $3.34 \mathrm{E}-02$ \\
Pgr025860 & 3.61 & 10.54 & 1.41 & 1.32 \\
Pgr000397 & 3.59 & 9.81 & 1.30 & $1.03 \mathrm{E}-04$ \\
Pgr010803 & 57.04 & 147.54 & 1.18 & $4.81 \mathrm{E}-04$ \\
Pgr000447 & 10.87 & 26.70 & & $7.87 \mathrm{E}-03$ \\
\hline
\end{tabular}

TPM, transcripts per million reads; FC, fold change; Adjusted $P$ value, $P$ value adjusted for multiple testing

mismatch and a 6-bp deletion in a PgUGT84A23 allele in sgRNA23+sgRNA24-1 (line 321) (Table 2).

\section{Knockout of PgUGT84A23 and PgUGT84A24 led to} changes in galloylglucose conjugates and derivatives in pomegranate hairy roots

To investigate the effect of the PgUGT84A23 and/or PgUGT84A24 mutations, phenolic metabolites in the control and mutant (ugt84a23, ugt84a24, ugt84a23 ugt84a24) hairy roots were analyzed (Fig. 2a). Eliminating PgUGT84A23 or PgUGT84A24 individually did not affect the metabolite profile significantly compared to the controls. However, when both UGT activities were abolished, punicalagins showed a $40 \%$ reduction in ugt84a23 ugt84a24 (Fig. 2a, c). Moreover, three new peaks (peaks 1-3) appeared in the dual CRISPR/Cas9-edited lines ugt84a23 ugt84a24 (Fig. 2a). The retention times and absorption spectra of peaks 1 and 2 matched those of the gallic acid 3-O- and 4$\mathrm{O}$-glucoside standards, respectively (Fig. 2a, b). Mass spectrometric (MS) analysis of peaks 1 and 2 confirmed that both compounds are conjugates of gallic acid and glucose $\left([\mathrm{M}-\mathrm{H}]^{-}\right.$at $m / z 331.07$ ) (Fig. 2b). In contrast to peaks 1 and 2 that were present in all of the ugt84a23 ugt84a24 lines, peak 3 (Fig. 2a, b, unidentified) was only detectable in twothirds of the ugt84a23 ugt84a24 hairy roots.

\section{A regiospecific gallic acid 4-O-glycosyltransferase was} discovered from transcriptome analysis of the CRISPR/ Cas9-edited hairy roots and biochemical characterization

To identify the UGT activities that produce gallic acid glucosides in ugt84a23 ugt84a24, transcriptome analysis was conducted on the control (three independent lines) and the dual edited hairy roots (lines 324, 327, 328, and 346; Table 2). Twelve UGTs showed significantly increased expression (greater than two-fold) in the ugt84a23 ugt84a24 lines compared to the controls (Table 3). Real-time quantitative polymerase chain reaction (qPCR) analysis confirmed that the transcript levels of 11 UGTs were higher in the ugt84a23 ugt84a24 lines than the controls (Fig. 3). Interestingly, the expression of Pgr008782 was also increased in the single CRISPR/Cas9edited lines ugt84a23 and ugt84a24 (Fig. 3). These 11 candidate UGTs were expressed as recombinant proteins in Escherichia coli and the purified proteins were assayed with gallic acid and UDP-glucose as substrates. Of the 11 recombinant UGTs, only PgUGT72BD1 was active toward 


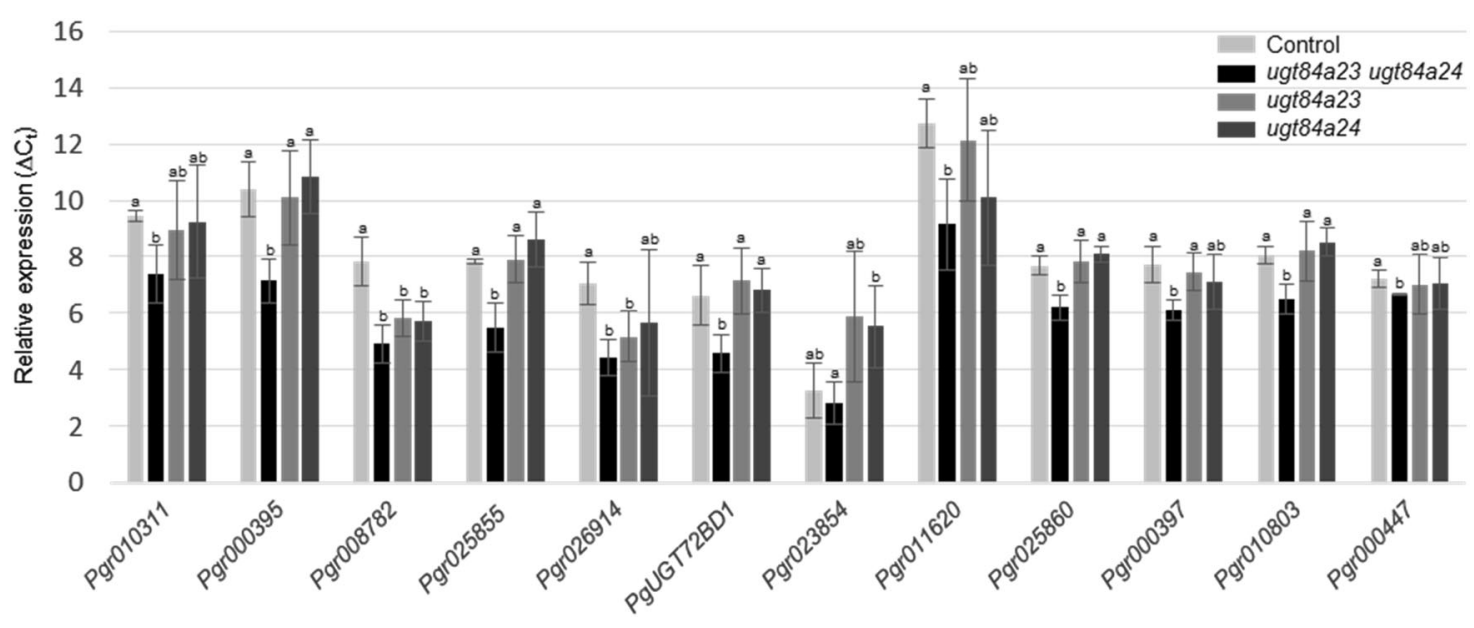

Fig. 3 Real-time qPCR analysis of the candidate UGTs in the control and mutant (ugt84a23, ugt84a24, ugt84a23 ugt84a24) hairy roots. The relative expression is indicated by the $\Delta C_{\mathrm{t}}$ value between the target gene and the housekeeping PgActin gene. Different letters indicate significant differences $(P<0.05)$ in the expression of the target gene

gallic acid and formed a single product, gallic acid 4-Oglucoside (Fig. 4a, c). The steady-state kinetics of $P g$ UGT72BD1 showed that it had a relatively high affinity to gallic acid $\left(K_{\mathrm{m}}=0.19 \pm 0.07 \mathrm{mM}\right)$ but a slow turnover $\left.\left[k_{\mathrm{cat}}=(2.83 \pm 0.5) \times 10^{-3} \mathrm{~s}^{-1}\right)\right]$ and low catalytic efficiency $\left[k_{\text {cat }} / K_{\mathrm{m}}=(0.15 \pm 0.03) \times 10^{-1} \mathrm{mM}^{-1} \mathrm{~s}^{-1}\right]$ (Fig. $\left.4 \mathrm{c}\right)$.

As with other plant UGTs, PgUGT72BD1 also contains a conserved PSPG motif at the C-terminus of the protein (Fig. 4b). Signal peptides were not detected in PgUGT72BD1, suggesting its localization in the cytosol (Fig. 4b). A phylogenetic analysis of representative UGTs placed PgUGT72BD1 in group E, together with $A t \mathrm{UGT71B1}, A t \mathrm{UGT72B1}, A t \mathrm{UGT71C1}$, and $A t \mathrm{UGT71C4}$, the UGTs that were previously shown with $3-O$ or 4-Oglucosylation activities toward hydroxybenzoic acids (gallic acid was not tested as a substrate for these UGTs) (Fig. 5) ${ }^{11}$. When the protein sequences of PgUGT72BD1, $A t \mathrm{UGT71B1}$, AtUGT72B1, AtUGT71C1, and AtUGT71C4 were compared, six amino acid sites were identified that are common among the 3-O (AtUGT72B1, At UGT71C1, and $A t \mathrm{UGT71C4})$ or $4-O$ (PgUGT72BD1 and $A t \mathrm{UGT71B1)}$ UGTs but differ between the two groups (Fig. 4b).

To understand the expression of PgUGT72BD1 in different pomegranate tissues, its transcript levels were initially determined by real-time qPCR. However, with the exception of roots $\left(C_{\mathrm{t}}\right.$ values around 27$)$, the $C_{\mathrm{t}}$ values obtained from other tissues were $>38$, suggesting a very low abundance of PgUGT72BD1 transcripts in stems, leaves, flowers, and fruit peels. Semi-qPCR was subsequently conducted, and consistent with the results from the real-time qPCR analysis, amplification products of PgUGT72BD1 were only detected in the root tissue (Fig. 4d). In contrast, PgUGT84A23 and PgUGT84A24. were expressed in all tissues examined (Fig. $4 \mathrm{~d}$ ).

\section{Discussion}

This study demonstrates that CRISPR/Cas9-based genome editing, A. rhizogenes-mediated hairy root transformation and non-destructive screening of transgenic hairy roots, as well as transcriptome analysis collectively enable efficient and effective gene discovery in pomegranate. The unique accumulation of gallic acid glucosides in the ugt84a23 ugt84a24 hairy roots also suggests that the CRISPR/Cas9 method holds the potential for developing new pomegranate cultivars with modified phytochemical profiles. The presence of gallic acid 3-Oand 4-O-glucosides has only been reported in fruits of blackcurrant, gooseberry, jostaberry, raspberry, blackberry, blueberry, Arbutus unedo (strawberry tree), and grape (pomace) ${ }^{12-14}$. Therefore, it is interesting that gallic acid glucosides can also be found in a non-reproductive plant tissue.

The CRISPR/Cas9-sgRNAs generated mismatches, inframe (3n, e.g., 3-bp, 6-bp), or out-of-frame (e.g., 1-bp, 4bp, 5-bp) deletions, as well as insertions of 1-bp, 2-bp, or 7-bp in PgUGT84A23 and PgUGT84A24 (Tables 1 and 2). The above-mentioned insertions and the out-of-frame deletions are expected to result in a frameshift and incorrect translation of the protein. Because the mutant lines containing in-frame deletions (removal of amino acids) exhibited metabolic phenotypes similar to those with insertions and out-of-frame deletions, it suggests that the missing amino acids resulting from the in-frame deletions play important roles in enzyme activities. In addition to the homozygous, monoallelic, and biallelic mutants, chimeras of more than two mutated alleles were also identified in the CRISPR/Cas9-edited hairy roots (Table 2). It could be because the hairy roots induced at the inoculation sites contain heterogeneous 
(a) (b)

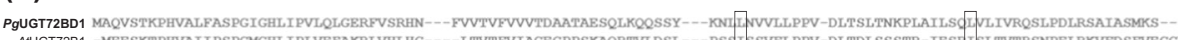

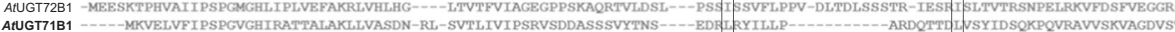
ATUGT71C1 -MGKQEDAELVII PFPFSGHTLATIELAKRLISQDNPRIHTITILYWGLPFIPQADTIAFLRSLVKNEPFTRLVTLPEVQDPP PMELFVVEFAES YILEYVKKMVPIIREALSTLLSS-RD AIUGT71C4 --MVKETELIFI PVPSTGHILVHIEFAKRLINLDH-RIHTITILNLSSPSSPHAS--VFARSLIASQPWERLHDLPPIQDPPPFDLYQRAPEA. IVKLIKKNTPLIKDAVSSIVASRRC

Un In M Purified

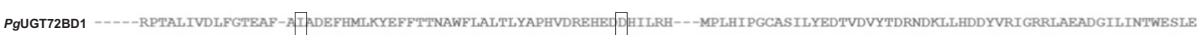

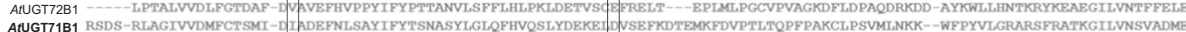

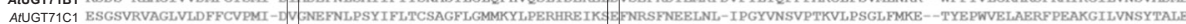
ATUGT71C4 GSDSVQVAGLVLDLFCNSLVKDVGNELNLPSYIYLTCNARYLGMMKYIPDRHRKIA E EFDLSS-GDEELPVPGFINAIPTKFMPPGLFNKE--AYEAYVELAPRFADAKGILVNSFTELE

PgUGT72BD1 PKTLQAT-RDPKAFGRF SQVRTLPIGPLVSGFQ---SFGSSQPKDDILQWLDDQPTESVLYVSFGSGGTLSADQTTELAWGLEQSQHRFIWVVRP PEENSACGAYFEQAKRADGTPEYLP AIUGT72B1 PNAIKAL-QEPGLDK----PPVYPVGPLVNIGK---QEAKQTEESECLKWLDNQPLGSVLYVSFGSGGTLTCEQLNELALLGLADSERRFLWVIRSPS-GIANSSYFDSH SQTD-PLTFLL

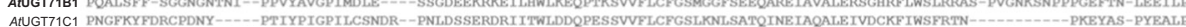

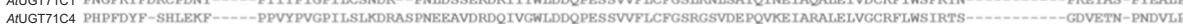

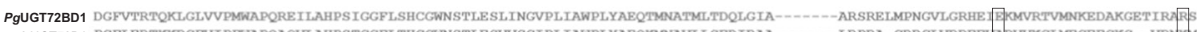

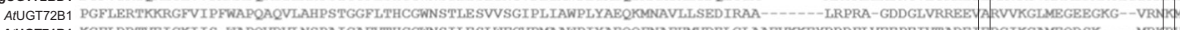
AtUGT71 K1 KOHER

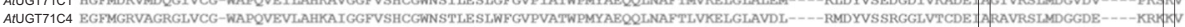

PgUGT72BD1 QDLKRTALLAVAEGGSSYAALSQVAQEECKTGLVGLKRRARGA

AIUGT72B1 KELKEAACRVLKDDGTSTKALSLVALKWKKAHKKELEQNGNH-

AUUGT71B1 MEMKDKLHVALVDGGSSNCALKKRPVQDVV-DNVP---- --

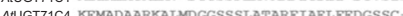

(c)

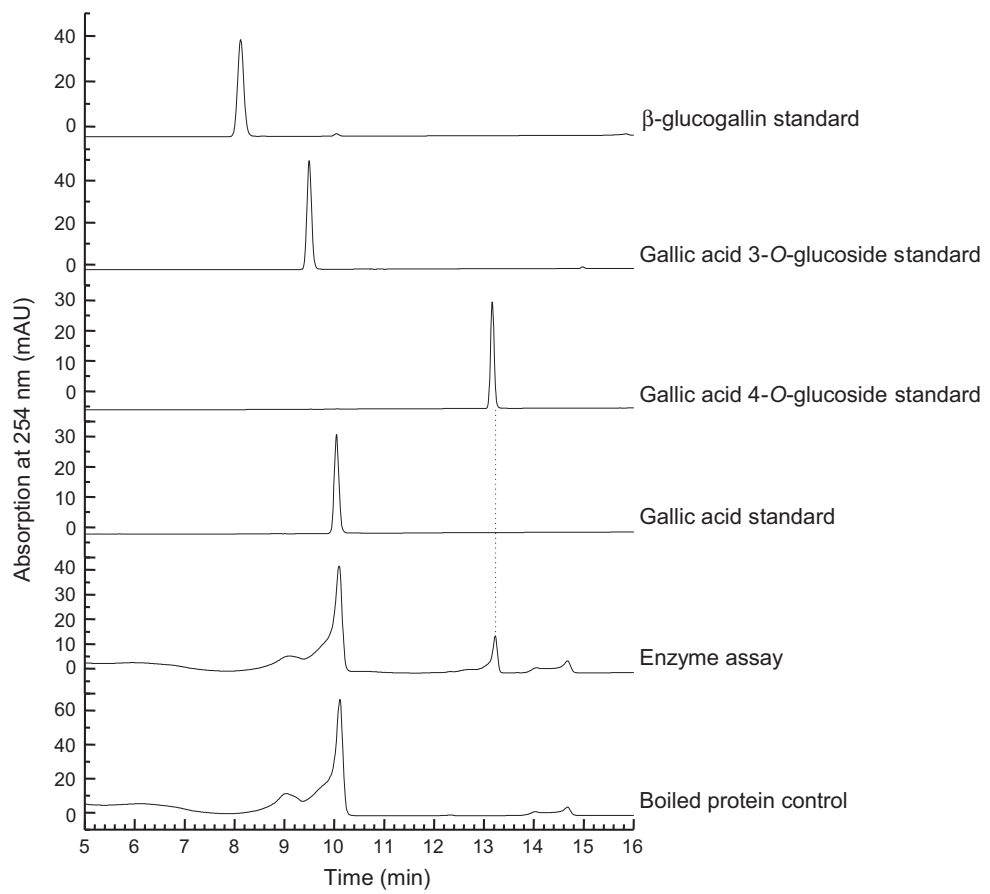

\begin{tabular}{|l|c|c|c|c|}
\hline & $K_{\mathrm{m}}(\mathrm{mM})$ & $V_{\max }\left(\mu \mathrm{mol} \mathrm{s} \mathrm{s}^{-1}\right)$ & $k_{\text {cat }}\left(\mathrm{s}^{-1}\right)$ & $k_{\text {cal }} / K_{\mathrm{m}}\left(\mathrm{mM}^{-1} \mathrm{~s}^{-1}\right)$ \\
\hline PgUGT72BD1 & $0.19 \pm 0.07$ & $(0.49 \pm 0.07) \times 10^{-1}$ & $(2.83 \pm 0.5) \times 10^{-3}$ & $(0.15 \pm 0.03) \times 10^{-1}$ \\
\hline
\end{tabular}

(d)

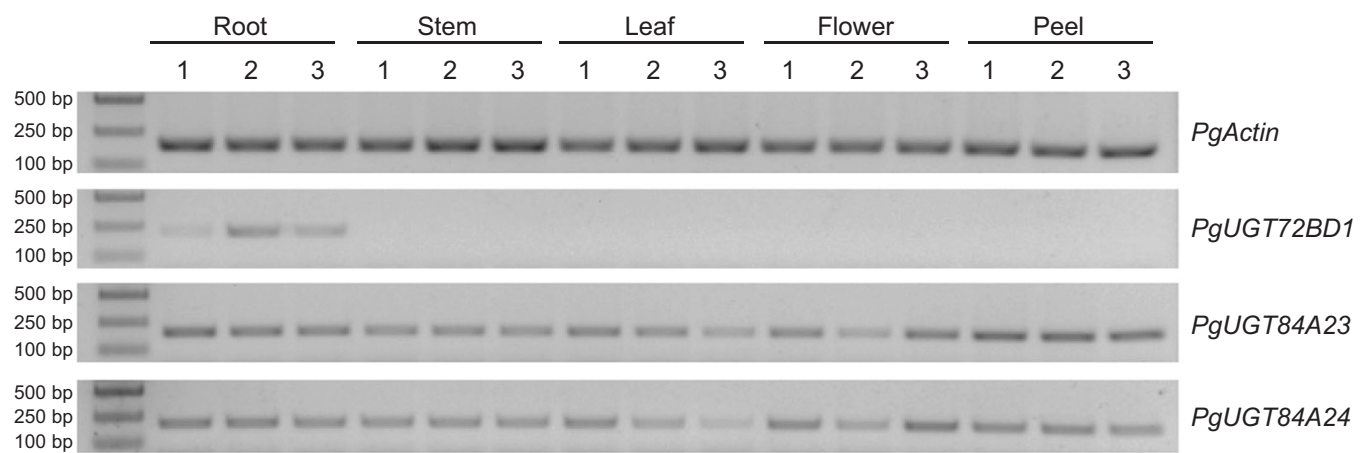

Fig. 4 (See legend on next page.) 
(see figure on previous page)

Fig. 4 Molecular and biochemical characterization of PgUGT72BD1. a Expression and purification of the recombinant PgUGT72BD1 protein in E. coli. The purified recombinant PgUGT72BD1 is indicated by an arrow. Un, uninduced; In, induced; M, protein molecular weight marker. b Multisequence alignment of PgUGT72BD1 and the group E UGTs previously shown to catalyze 3-O- or 4-O-glucosylation of hydroxybenzoic acid substrates. UGTs capable of 4-O-glucosylation are highlighted in bold. Amino acids that are common among 3-O or 4-O UGTs but differ between the two groups are indicated with boxes. The conserved Plant Secondary Product Glycosyltransferase (PSPG) motif is underlined. c PgUGT72BD1 produces gallic acid 4-O-glucoside from gallic acid and UDP-glucose. The kinetic parameters of PgUGT72BD1 toward gallic acid are shown. $\mathbf{d}$ Tissuespecific expression of PgUGT72BD1, PgUGT84A23, and PgUGT84A24. Total RNA was extracted from three biological replicates (1-3) of each pomegranate tissue type and used for reverse transcription (RT). The RT products served as templates for the semi-quantitative PCR reactions

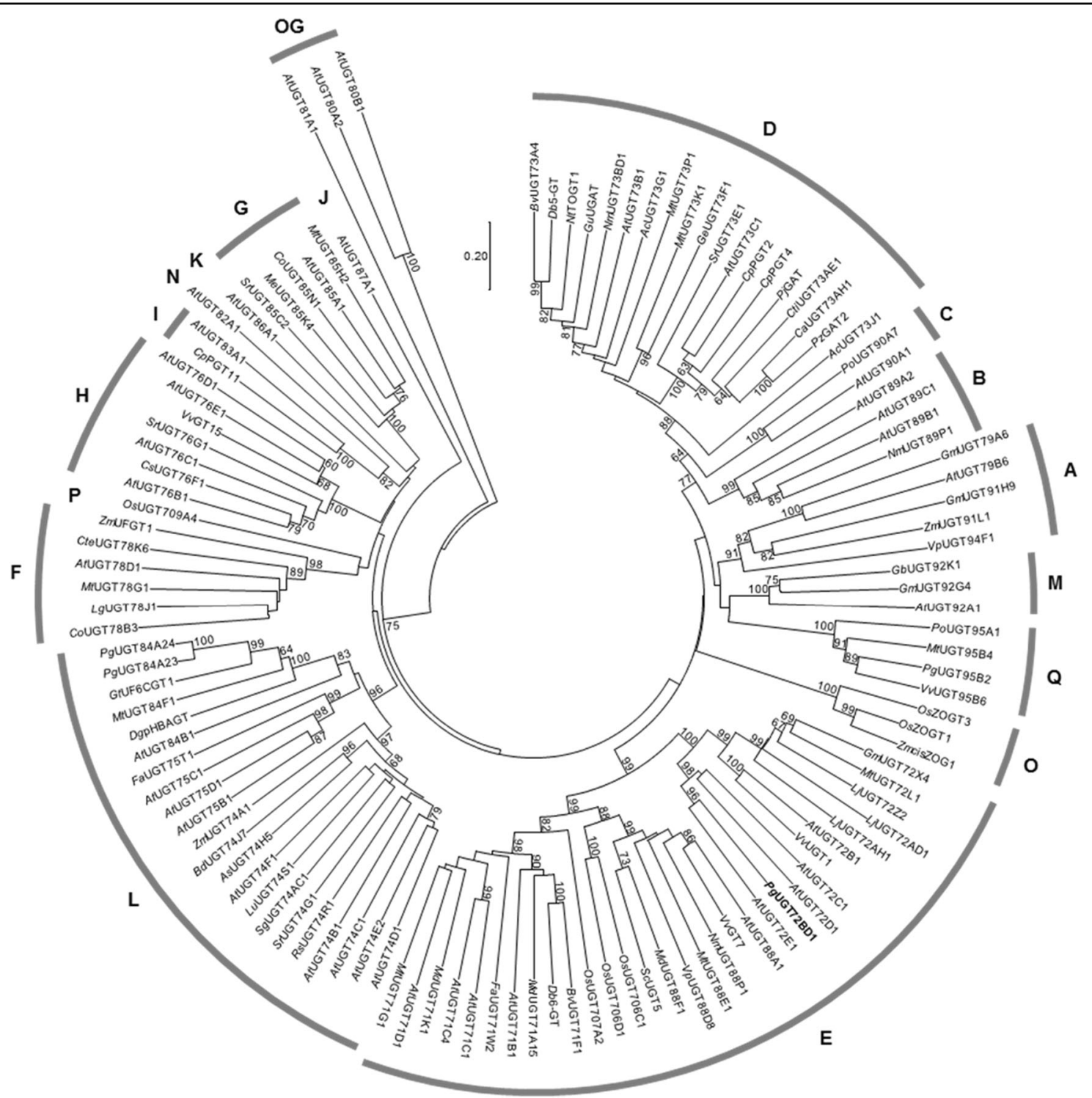

Fig. 5 Phylogeny of functionally characterized pomegranate UGTs and selected UGTs representing different plant UGT phylogenetic groups. PgUGT72BD1 is highlighted in bold. Bootstrap values $>60$ are shown next to the branches. OG, outgroup

cells of different CRISPR/Cas9-edited or non-edited gene alleles. To establish homogeneous hairy root clones, a single hairy root tip would need to be recultured in phytohormone-free growth medium for multiple rounds and then tested for homogeneity. On the other hand, the metabolic phenotype of ugt $84 a 23$ 
ugt84a24 hairy roots (appearance of new peaks) indicates the advantage of metabolite profiling in detecting knockout of enzyme activities in a heterogeneous cell population.

Eliminating both $P g \mathrm{UGT} 84 \mathrm{~A} 23$ and PgUGT84A24 activities frees gallic acid from the biosynthesis of $\beta$-glucogallin (Fig. 1a). The transitory increase in the cellular gallic acid concentration may regulate the expression/activity of UGT(s) that convert gallic acid to the glucoside derivatives (Fig. 1a). Indeed, gallic acid 3-Oand 4-O-glucosides accumulated in the ugt84a23 ugt84a24 hairy roots (Fig. 2). In addition, transcriptome and real-time qPCR analyses identified 11 UGTs with increased expression in ugt84a23 ugt84a24 and one of the candidate UGTs, PgUGT72BD1, exhibited regioselective glucosylation of gallic acid at the 4-OH position (Table 3; Figs. 3 and 4). However, none of the candidate UGTs produced gallic acid 3-O-glucoside, suggesting that the gallic acid 3-O-glucosylation activity may be regulated at a level other than transcription.

$P g$ UGT72BD1, $P g$ UGT84A23, and PgUGT84A24 can all use gallic acid as substrate but form galloylglucose conjugates with ether or ester linkages (Fig. 1a). PgUGT72BD1 has a higher affinity to gallic acid $\left(K_{\mathrm{m}}=\right.$ $0.19 \pm 0.07 \mathrm{mM}$; Fig. 4c) than PgUGT84A23 $\left(K_{\mathrm{m}}=0.89 \pm\right.$ $0.07 \mathrm{mM})$ and PgUGT84A24 $\left(K_{\mathrm{m}}=0.98 \pm 0.01 \mathrm{mM}\right)^{10}$. However, the turnover numbers of PgUGT84A23 $\left(k_{\text {cat }}=\right.$ $\left.0.52 \pm 0.03 \mathrm{~s}^{-1}\right)$ and PgUGT84A24 $\left(k_{\mathrm{cat}}=0.55 \pm 0.01 \mathrm{~s}^{-1}\right)$ are $>180$-fold higher than that of PgUGT72BD1 $\left[k_{\text {cat }}=\right.$ $\left.(2.83 \pm 0.5) \times 10^{-3} \mathrm{~s}^{-1}\right]$. As a result, the catalytic efficiency of PgUGT72BD1 $\left[k_{\text {cat }} / K_{\mathrm{m}}=(0.15 \pm 0.03) \times 10^{-1} \mathrm{mM}^{-1} \mathrm{~s}^{-1}\right.$ ] is about 38 -fold lower than that of $\operatorname{PgUGT} 84 \mathrm{~A} 23\left(k_{\mathrm{cat}} / K_{\mathrm{m}}=\right.$ $\left.0.58 \mathrm{mM}^{-1} \mathrm{~s}^{-1}\right)$ and PgUGT84A24 $\left(k_{\mathrm{cat}} / K_{\mathrm{m}}=0.56 \mathrm{mM}^{-1} \mathrm{~s}^{-1}\right)$ (Fig. 4c) ${ }^{10}$. Therefore, even though PgUGT72BD1 is expressed in the wild-type pomegranate roots and hairy roots, gallic acid is mainly used for the biosynthesis of $\beta$-glucogallin (and HTs) by PgUGT84A23 and PgUGT84A24 due to their much higher catalytic efficiencies than $P g \mathrm{UGT72BD} 1$. Indeed, our previous metabolite profiling analysis did not identify gallic acid 4-Oglucoside in any pomegranate tissues ${ }^{10}$. These results also suggest that the primary role of PgUGT72BD1 in pomegranate roots could be glycosylating aglycones other than gallic acid. Intriguingly, HT production was not completely abolished in ugt84a23 ugt84a24 (Fig. 2), suggesting that there could be additional UGT(s) contributing to $\beta$-glucogallin formation in pomegranate.

Of the 17 so far defined UGT phylogenetic groups (A-Q) in plants, PgUGT72BD1 (gallic acid 4-O UGT) belongs to group E that contains UGT71, UGT72, and UGT88 gene families (Fig. 5). Regioselective glycosylation of hydroxybenzoic acids (structurally similar to gallic acid) was previously reported for members of group E UGTs, including $A t \mathrm{UGT} 71 \mathrm{~B} 1$ that only glycosylates the $4-\mathrm{OH}$ position and $A t \mathrm{UGT} 71 \mathrm{C} 1, A t \mathrm{UGT71C4}$, and $A t \mathrm{UGT72B} 1$ that specifically glycosylate the $3-\mathrm{OH}$ position ${ }^{11}$. Six amino acids are conserved in the hydroxybenzoic acid/ gallic acid 3-O or 4-O UGTs but distinct between the two groups of regioselective UGTs (Fig. 4b). The function of these amino acids in determining the regioselectivity of the corresponding UGTs can be explored by site-directed mutagenesis and enzyme assays. In addition, once the gallic acid 3-O UGT is cloned in pomegranate, the protein sequences and structural features of the gallic acid 3-O and 4-O UGTs can be compared to identify the key amino acid(s) for regioselectivity. Furthermore, it was proposed that the regioselectivity for hydroxycoumarins (a group of phenolic metabolites) was switched among the UGT71, UGT72, and UGT88 families during the evolution of group $\mathrm{E} \mathrm{UGTs}{ }^{15}$. It will be interesting to understand whether regioselectivity switching event(s) for gallic acid also occurred among these UGT gene families.

\section{Conclusions}

In this work, the CRISPR/Cas9-mediated editing of two galloylglucose ester-forming UGTs, PgUGT84A23 and $\operatorname{PgUGT84A24}$, in pomegranate hairy roots generated various mismatches, insertions, and deletions in the target genes. Metabolite analysis of the transgenic hairy roots showed modified phenolic profiles, particularly the accumulation of 3-O- and 4-O-glucosides of gallic acid, in the ugt84a23 ugt84a24 double mutant lines. Transcriptome and real-time qPCR analyses identified multiple UGTs with increased expression in the ugt84a23 ugt84a24 hairy roots compared to the vectortransformed controls. Biochemical characterization of the candidate genes discovered a group E UGT (PgUGT72BD1) that glycosylates specifically the 4-O position of gallic acid.

The pomegranate genome has recently been sequenced, providing an exciting opportunity for exploring this ancient fruit and modern functional food ${ }^{16,17}$. Together with genome, transcriptome, and metabolite analyses, the CRISPR/Cas9 method renders functional genomics in pomegranate, a woody plant and non-traditional model system, more accessible. In addition, building a genomeediting platform in pomegranate will also facilitate germplasm improvement as well as the sustainable development of pomegranate as a horticultural crop and functional food.

\section{Materials and methods}

Construction of expression plasmids for Cas9 and sgRNAs

The sgRNAs for PgUGT84A23 and PgUGT84A24 were designed using Cas-Designer (http://www.rgenome.net/casdesigner $/)^{18}$; those with high-quality scores were then subjected to testing for secondary structures using the mfold web server (http://mfold.rna.albany.edu/?=mfold/RNA-Folding- 
Form2.3) ${ }^{19}$. The following sgRNAs were selected in this study: sgRNA23 for PgUGT84A23 (5'-GGGTCAAGGGCACGTGAAC-3') and two sgRNAs, sgRNA24-1 (5'-GG GAGGAGCCGTCGCCTAT-3') and sgRNA24-2 (5'-ATC CCGTGGGTGTCTGACG-3'), for PgUGT84A24.

The sgRNAs were cloned into the psgR-Cas9-At backbone, which contains the AtU6 promoter for the expression of the sgRNA as well as the $A t U B Q 1$ promoter and terminator for the expression of $S p \mathrm{Cas}^{20}$. For easy identification of transgenic hairy roots, the hygromycin B resistance gene in the plant transformation vector pCAMBIA1300 was replaced with a GFP gene and the resulting plasmid vector was designated pCAMBIA1300GFP. The sgRNA and Cas9 containing psgR-Cas9-At cassette was cloned into the EcoRI and HindIII sites of pCAMBIA1300-GFP. Because a high-quality sgRNA targeting both PgUGT84A23 and PgUGT84A24 was not identified, the sgRNAs for PgUGT84A23 and PgUGT84A24 were cloned into the p2xsgR-Cas9-At cassette (i.e., sgRNA23+sgRNA24-1 or sgRNA23+sgRNA24-2) where the two sgRNAs were each directed by an AtU6 promoter. The resulting cassettes were cloned into the EcoRI and HindIII sites of pCAMBIA1300-GFP.

\section{Agrobacterium rhizogenes-mediated induction and transformation of pomegranate hairy roots}

The sgRNA and Cas9-expressing pCAMBIA1300-GFP plasmids and the empty pCAMBIA1300-GFP vector were transformed into the A. rhizogenes strain MSU440 through electroporation. Induction and transformation of pomegranate hairy roots using the hypocotyl explants were carried out as described ${ }^{9}$. The hairy roots were observed under a fluorescent microscope (Leica DM6000B, Leica Microsystems, Wetzlar, Germany) after 21 days. Hairy roots that emitted green fluorescence upon excitation were marked on the plate and the non-green fluorescent hairy roots were removed using a scalpel. Only one green fluorescent hairy root was maintained on each plate. These hairy roots were transferred to plates containing fresh growth media every 3 weeks. After about 2 months, the hairy root tissue was collected, frozen in liquid nitrogen, and ground into fine powder using mortar and pestle.

\section{Detection of CRISPR/Cas9-mediated gene editing}

To identify the CRISPR/Cas9-edited PgUGT84A23 and PgUGT84A24 alleles, genomic DNA was extracted from transgenic hairy roots using a Cetyltrimethyl Ammonium Bromide-based method ${ }^{21}$. PCR reactions were performed using the genomic DNA as template and PgUGT84A23F (5'-GTTCGGAGTCGTCACTTGTC-3') and PgUGT84A23R (5'-ATCTCGTGCTCAAGTTCCTG-3') for amplification of the PgUGT84A23 alleles and
PgUGT84A24F (5'-GGGGTCCGAGTCGTTGGTTC-3') and PgUGT84A24R (5'-GCACGGCAACTGGACATCG$\left.3^{\prime}\right)$ for the PgUGT84A24 alleles. The PCR products were analyzed directly by DNA sequencing. The DNA sequence chromatograms of the homozygous wild-type or mutant alleles showed individual, evenly distributed peaks. When a mixture of different mutant alleles or a combination of wild-type and mutant alleles was present in the PCR products, the chromatograms displayed overlapping peaks. In the latter case, the PCR products were cloned into a TA cloning vector pMD19-T (Takara Biomedical Technology Co., Ltd., Beijing, China). The resulting plasmids were transformed into $E$. coli $\mathrm{DH} 5 \alpha$ cells and multiple colonies were selected for plasmid preparation and DNA sequencing.

\section{Metabolite analysis of transgenic hairy roots}

The ground hairy root tissue was extracted in 70\% methanol for $60 \mathrm{~min}$ under sonication and centrifuged at $13,000 \mathrm{rpm}$ for $10 \mathrm{~min}$. The supernatant was transferred to an high-performance liquid chromatography (HPLC) vial and $20 \mu \mathrm{L}$ was injected onto an Agilent 1200 HPLC. Metabolite separation was performed using a reversephase $\mathrm{C}_{18}$ column (Diamonsil, $250 \mathrm{~mm} \times 4.6 \mathrm{~mm}$, particle size $5 \mu \mathrm{m})$ and a previously established gradient ${ }^{22}$. Peaks of interest were collected from multiple HPLC runs, pooled, concentrated, and subjected to high-resolution electrospray ionization MS analysis as described ${ }^{22}$. The gallic acid, gallic acid 3-O-glucoside, and gallic acid 4-Oglucoside standards were purchased from ZZBIO Co. LTD (Shanghai, China). One-way analysis of variance (ANOVA) followed by Tukey's honestly significant difference (HSD) post hoc test was conducted on the peak areas of punicalagins (punicalagin $\alpha$ and $\beta$ isomers) using JMP 14.2.0 (SAS Institute, 2018).

\section{Transcriptome analysis of transgenic hairy roots}

Three vector-transformed and four ugt84a23 ugt84a24 hairy root lines (Table 2) were selected for comparative transcriptome analysis. Total RNA was extracted from hairy roots using Trizol reagent (Invitrogen, Carlsbad, CA). RNAseq libraries were constructed using the Illumina TruSeq RNA Sample Prep Kit and subjected to sequencing on an Illumina HiSeq4000, with about 60 million paired-end reads $(2 \times 150 \mathrm{bp})$ per sample. The raw reads were cleaned to remove the adapter sequences as well as short or low-quality sequences using SeqPrep (https://github.com/jstjohn/SeqPrep) and Sickle (https:// github.com/najoshi/sickle). The trimmed reads were mapped to the pomegranate genome using Hisat2 to obtain read counts ${ }^{23}$. The read counts were quantified by the RNA-Seq by Expectation Maximization method and expressed as transcripts per million reads ${ }^{24}$. The 
differential gene expression analysis between the controls and ugt84a23 ugt84a24 lines was performed using DESeq2, with adjusted $P$ value $<0.05$ and $\left|\log _{2} \mathrm{FC}\right| \geq 1^{25}$. The transcriptome data were deposited in the NCBI sequence reads archive under the accession PRJNA550088.

\section{Expression and purification of recombinant proteins and UGT enzyme assays}

The open reading frames of candidate UGTs were codon optimized for E. coli expression, synthesized by Genewiz (Suzhou, China), and cloned into the pET28a vector. The recombinant plasmids were transformed into E. coli BL21 (DE3) and the cells were grown at $37^{\circ} \mathrm{C}$ in the Luria Bertani media until $\mathrm{OD}_{600}$ reached 0.8. Protein expression was induced by adding isopropyl $\beta$-D-1-thiogalactopyranoside to a final concentration of $0.1 \mathrm{mM}$. The cells were grown at $17^{\circ} \mathrm{C}$ for an additional $18 \mathrm{~h}$ and harvested by centrifugation. The cell pellets were resuspended in the lysis buffer (50 mM MES, pH 5.5, $300 \mathrm{mM}$ $\mathrm{NaCl}$, and $50 \mathrm{mM}$ imidazole) and homogenized using a cell disruptor (Constant Systems Ltd, Northants, UK). The His-tagged recombinant UGT proteins were purified using Ni-NTA resin (Thermo Fisher Scientific, Waltham, MA, USA). The concentration of the purified proteins was measured using the Bradford assay ${ }^{26}$. The purified protein was kept in the storage buffer [50 mM MES, pH 5.5, $100 \mathrm{mM} \mathrm{NaCl}$, and $10 \%(\mathrm{w} / \mathrm{v})$ glycerol] at $-80^{\circ} \mathrm{C}$.

For UGT enzyme assays, the $160-\mu \mathrm{L}$ reaction mixture contained $50 \mathrm{mM}$ MES, pH 5.5, $0.6 \mathrm{mM}$ UDP-glucose, $0.25 \mathrm{mM}$ gallic acid, $3 \mathrm{mM}$ 2-mercaptoethanol, and $1.6 \mu \mathrm{g}$ of purified protein. After incubating at $30^{\circ} \mathrm{C}$ for $12 \mathrm{~h}$, the reaction was terminated by adding $16 \mu \mathrm{L}$ of trifluoroacetic acid $(100 \%, \mathrm{w} / \mathrm{v})$ and $400 \mu \mathrm{L}$ of methanol. The reaction mixture was filtered through a $0.22-\mu \mathrm{m}$ filter and $70 \mu \mathrm{L}$ of the flow-through was injected onto an Agilent 1200 HPLC with a reverse-phase $\mathrm{C}_{18}$ column (YMC-Pack ODS-AQ $\mathrm{C}_{18} \mathrm{~L} 1,250 \mathrm{~mm} \times 4.6 \mathrm{~mm}$, particle size $5 \mu \mathrm{m}$ ). The elution gradient was between (A) $0.1 \%$ formic acid in water and (B) acetonitrile at $0-3 \mathrm{~min}, 3 \% \mathrm{~B} ; 3-5 \mathrm{~min}, 3-5 \% \mathrm{~B}$; 5-15 min, $5-15 \%$ B; $15-16 \mathrm{~min}, \quad 15-60 \% \quad \mathrm{~B}$; and 16-18 min, $60-3 \% \mathrm{~B}$. The flow rate was $1 \mathrm{~mL} \mathrm{~min}^{-1}$. The kinetic analysis of $P g \mathrm{UGT} 72 \mathrm{BD} 1$ with gallic acid as substrate was performed as previously described ${ }^{27}$, with slight modifications. The gallic acid concentrations were between 30 and $500 \mu \mathrm{M}$ and the reactions were incubated at $30^{\circ} \mathrm{C}$ for 45,75 , and $135 \mathrm{~min}$.

\section{Reverse transcription (RT) (semi)-qPCR analysis}

Total RNA was extracted from transgenic hair roots or pomegranate leaf, stem, root, flower, and fruit peel tissues using the RNAprep Pure Plant Kit (Tiangen Biotech Co., Ltd., Beijing, China) and used for RT with the PrimeScript $^{\mathrm{TM}}$ RT Reagent Kit (Takara). qPCR was performed using the TB Green Premix Ex Taq ${ }^{\mathrm{TM}}$ (Tli RNaseH Plus) Kit (Takara) on a StepOnePlus Real-Time PCR System (Thermo Fisher Scientific). Melting curve analysis was conducted immediately after the PCR reactions and only one product was observed for each primer pair. The RTqPCR reactions were performed using three biological replicates, each with three technical replicates. The expression levels of candidate UGTs in the control and mutant (ugt84a23, ugt84a24, ugt84a23 ugt84a24) samples were presented using $\Delta C_{\mathrm{t}}$ values $\left(C_{\mathrm{t}}\right.$ PgUGT $-C_{\mathrm{t}}$ PgActin $)$. Statistical analysis was conducted using ANOVA and Tukey's HSD test in JMP 14.2.0 (SAS Institute). The primer sequences, amplicon sizes, and amplification efficiencies are shown in Table S1.

For the semi-qPCR analysis, $1 \mu \mathrm{L}$ of the first-strand cDNA was used as template for amplification by TaKaRa Taq DNA polymerase (Takara) and primers specific for PgUGT72BD1, PgUGT84A23, PgUGT84A24, or PgActin (Table S1). The PCR conditions were as follows: $94{ }^{\circ} \mathrm{C}$ for 5 min, followed by 25 cycles (PgActin, PgUGT84A23, PgUGT84A24) or 30 cycles (PgUGT72BD1) of $94{ }^{\circ} \mathrm{C}$ for $30 \mathrm{~s}, 60^{\circ} \mathrm{C}$ for $30 \mathrm{~s}$, and $72{ }^{\circ} \mathrm{C}$ for $45 \mathrm{~s}$, and a final extension step of $72{ }^{\circ} \mathrm{C}$ for $10 \mathrm{~min}$. The PCR products were analyzed on a $1.5 \%$ agarose gel.

\section{Phylogenetic analysis}

Alignment of plant UGT sequences was conducted using Multiple Sequence Comparison by Log-Expectation $\left(\right.$ MUSCLE) ${ }^{28}$. A neighbor-joining tree was built using Molecular Evolutionary Genetics Analysis and assessed with 1000 bootstrap replicates ${ }^{29}$. The AGI (Arabidopsis sequences) and GenBank (non-Arabidopsis sequences) accession numbers for the UGTs are: AcUGT73G1 (AAP88406), AcUGT73J1 (AAP88407), AsUGT74H5 (ACD03250), AtUGT71B1 (AT3G21750), AtUGT71C1 (AT2G29750), AtUGT71C4 (AT1G07250), AtUGT71D1 (AT2G29730), AtUGT72B1 (AT4G01070), AtUGT72C1 (AT4G36770), $A t \mathrm{UGT72D1}$ (AT2G18570), AtUGT72E1 (AT3G50740), $A t \mathrm{UGT73B1}$ (AT4G34138), AtUGT73C1 (AT2G36750), AtUGT74B1 (AT1G24100), AtUGT74C1 (AT2G31790), $A t \mathrm{UGT74D1}$ (AT2G31750), AtUGT74E2 (AT1G05680), $A t \mathrm{UGT74F1}$ (AT2G43840), AtUGT75B1 (AT1G05560), AtUGT75C1 (AT4G14090), AtUGT75D1 (AT4G15550), $A t \mathrm{UGT76B1}$ (AT3G11340), AtUGT76C1 (AT5G05870), $A t \mathrm{UGT76D1}$ (AT2G26480), AtUGT76E1 (AT5G59580), $A t \mathrm{UGT78D} 1$ (AT1G30530), AtUGT79B6 (AT5G54010), AtUGT80B1 (AT1G43620), AtUGT80A2 (AT3G07020), AtUGT81A1 (AT4G31780), AtUGT82A1 (AT3G22250), AtUGT83A1 (AT3G02100), AtUGT84B1 (AT2G23260), AtUGT85A1 (AT1G22400), AtUGT86A1 (AT2G36970), AtUGT87A1 (AT2G30150), AtUGT88A1 (AT3G16520), AtUGT89A2 (AT5G03490), AtUGT89B1 (AT1G73880), AtUGT89C1 (AT1G06000), AtUGT90A1 (AT2G16890), $A t$ UGT92A1 (AT5G12890), BdUGT74J7 (XP_003581017), 
BvUGT71F1 (AY526081), BvUGT73A4 (AY526080), CaUGT73AH1 (AUR26623), CoUGT78B3 (AEB61484), CoUGT85N1 (AEB61489), CpPGT2 (AIS39471), CpPGT4 (AIS39473), CpPGT11 (AIS39477), CsUGT76F1 (KDO69246), CteUGT78K6 (BAF49297), CtiUGT73AE1 (AJT58578), Db5-GT (CAB56231), Db6-GT (AAL57240), DgpHBAGT (BAO66179), FaUGT71W2 (XP_011468178), FaUGT75T1 (XP_004307485), GbUGT92K1 (ASK39406), GeUGT73F1 (BAC78438), GmUGT72X4 (KRH46505), GmUGT79A6 (BAN91401), GmUGT91H9 (NP_001348424), GmUGT92G4 (KRH14708), GtUF6CGT (AB985754), GuUGAT (ANJ03631), LgUGT78J1 (AEB61487), LjUGT72AD1 (AP009657), LjUGT72AH1 (AOG18241), LjUGT72Z2 (AKK25344), LuUGT74S1 (AGD95005), MdUGT71A15 (AAZ80472), MdUGT71K1 (ACZ44835), MdUGT88F1 (ARV88476), MeUGT85K4 (AEO45781), MtUGT71G1 (AAW56092), MtUGT72L1 (ACC38470), $M t \mathrm{UGT73K1}$ (AAW56091), MtUGT73P1 (ABI94026), $\quad M t \mathrm{UGT78G1}$ (ABI94025), MtUGT84F1 (ABI94023), $\quad M t \mathrm{UGT} 85 \mathrm{H} 2$ (ABI94024), $\quad M t \mathrm{UGT} 88 \mathrm{E} 1$ (ABI94021), MtUGT95B4 (XP_003612636), NmUGT73BD1 (LC368259), NmUGT88P1 (CEO43476), NmUGT89P1 (LC368262), NtTOGT1 (AAB36653), OsUGT706C1 (BAB68090), OsUGT706D1 (BAB68093), OsUGT707A2 (BAC83994), OsUGT709A4 (BAC80066), OsZOGT1 (BAS90436), OsZOGT3 (BAS90518), PgUGT72BD1 (MN124519), PgUGT84A23 (ANN02875), PgUGT84A24 (ANN02877), PgUGT95B2 (MH507175), PjGAT (AYA60333), PoUGT90A7 (EU561019), PoUGT95A1 (ACB56927), PzGAT2 (AYA60331), RsUGT74R1 (ABP49574), ScUGT5 (BAJ11653), SgUGT74AC1 (AEM42999), SrUGT73E1 (AAR06917), SrUGT74G1 (AY345982), SrUGT76G1 (AAR06912), SrUGT85C2 (AAR06916), VpUGT88D8 (BAH47552), $V p$ UGT94F1 (BAI44133), $V v$ GT7 (XP_002276546), VvGT15 (XP_002281513), $V v$ UGT1 (CBI34463), VvUGT95B6 (XP_010664783), ZmUFGT1 (P16167), ZmUGT74A1 (NP_001105326), ZmUGT91L1 (NP_001347041), and ZmcisZOG1 (AAK53551). Ac, Allium cepa; As, Avena strigosa; At, Arabidopsis thaliana; Bd, Brachypodium distachyon; Bv, Beta vulgaris; Ca, Centella asiatica; $C$, Consolida orientalis; $C p$, Citrus paradise; $C s$, Citrus sinensis; Cte, Clitoria ternatea; Cti, Carthamus tinctorius; Db. Dorotheanthus bellidiformis; Dg, Delphinium grandiflorum; Fa, Fragaria $\times$ ananassa; Gb, Ginkgo biloba; Ge, Glycyrrhiza echinata; Gm, Glycine max; Gt, Gentiana triflora; Gu, Glycyrrhiza uralensis; Lg, Lamium galeobdolon; Lj, Lotus japonicus; Lu, Linum usitatissimum; Md, Malus $\times$ domestica; Me, Manihot esculenta; Mt, Medicago truncatula; Nm, Nemophila menziesii; Nt, Nicotiana tabacum; Os, Oryza sativa; Pg, Punica granatum; Pj, Panax japonicus; Po, Pilosella officinarum; Pz, Panax zingiberensis; Rs, Rhodiola sachalinensis; Sc, Sinningia cardinalis; Sg, Siraitia grosvenorii; Sr, Stevia rebaudiana; Vp, Veronica persica; $V v$, Vitis vinifera; Zm, Zea mays.

\section{Acknowledgements}

We thank Dr. Jiankang Zhu (Shanghai Center for Plant Stress Biology, Chinese Academy of Sciences) for providing us the psgR-Cas9-At and p2xsgR-Cas9-At plasmids. This work was supported by the Science and Technology Commission of Shanghai Municipality under grant 14DZ2260400 and the Special Fund for Scientific Research of Shanghai Landscaping and City Appearance Administrative Bureau under grants G172403 and G182403.

\section{Author details}

'Shanghai Key Laboratory of Plant Functional Genomics and Resources, Shanghai Chenshan Botanical Garden, 201602 Shanghai, China. ${ }^{2}$ Shanghai Chenshan Plant Science Research Center, Chinese Academy of Sciences, 201602 Shanghai, China. ${ }^{3}$ Department of Plant Sciences, University of California, Davis, CA 95616, USA

\section{Authors' contributions}

L.C. and L.T. conceived the study; L.C. and S.W. performed analysis; all authors analyzed the data and wrote the manuscript.

\section{Conflict of interest}

The authors declare that they have no conflict of interest.

Supplementary information accompanies this paper at (https://doi.org/ 10.1038/s41438-019-0206-7).

Received: 8 July 2019 Revised: 27 August 2019 Accepted: 1 October 2019 Published online: 08 November 2019

\section{References}

1. Wu, S. \& Tian, L. Diverse phytochemicals and bioactivities in the ancient fruit and modern functional food pomegranate (Punica granatum). Molecules 22, 1606 (2017).

2. Bar-Ya'akov, I., Tian, L., Amir, R. \& Holland, D. Primary metabolites, anthocyanins, and hydrolyzable tannins in the pomegranate fruit. Front. Plant Sci. 10,620 (2019)

3. Holland, D., Hatib, K. \& Bar-Ya'akov, I. in Horticultural Reveiws (ed. Janick, J.) 127-191 (John Wiley \& Sons, 2009).

4. Wang, H., Russa, M. L. \& Qi, L. S. CRISPR/Cas9 in genome editing and beyond. Annu. Rev. Biochem. 85, 227-264 (2016).

5. Ma, X., Zhu, Q., Chen, Y. \& Liu, Y.-G. CRISPR/Cas9 platforms for genome editing in plants: developments and applications. Mol. Plant 9, 961-974 (2016).

6. Koonin, E. V., Makarova, K. S. \& Zhang, F. Diversity, classification and evolution of CRISPR-Cas systems. Curr. Opin. Microbiol. 37, 67-78 (2017).

7. Knott, G. J. \& Doudna, J. A. CRISPR-Cas guides the future of genetic engineering. Science 361, 866-869 (2018).

8. Chen, K., Wang, Y., Zhang, R., Zhang, H. \& Gao, C. CRISPR/Cas genome editing and precision plant breeding in agriculture. Annu. Rev. Plant Biol. 70, 667-697 (2019).

9. Ono, N., Bandaranayake, P. C. G. \& Tian, L. Establishment of pomegranate (Punica granatum) hairy root cultures for genetic interrogation of the hydrolyzable tannin biosynthetic pathway. Planta 236, 931-941 (2012).

10. Ono, N., Qin, X., Wilson, A., Li, G. \& Tian, L. Two UGT84 family glycosyltransferases catalyze a critical reaction of hydrolyzable tannin biosynthesis in pomegranate (Punica granatum). PLOS ONE 11, e0156319 (2016).

11. Lim, E. et al. The activity of Arabidopsis glycosyltransferases toward salicylic acid, 4-hydroxybenzoic acid, and other benzoates. J. Biol. Chem. 277, 586-592 (2002).

12. Schuster, B. \& Herrmann, K. Hydroxybenzoic and hydroxycinnamic acid derivatives in soft fruits. Phytochemistry 24, 2761-2764 (1985).

13. Pawlowska, A. M., De Leo, M. \& Braca, A. Phenolics of Arbutus unedo L. (Ericaceae) fruits: identification of anthocyanins and gallic acid derivatives. J. Agric Food Chem. 54, 10234-10238 (2006).

14. Lu, Y. \& Foo, L. Y. The polyphenol constituents of grape pomace. Food Chem 65, 1-8 (1999). 
15. Lim, E.-K. et al. Evolution of substrate recognition across a multigene family of glycosyltransferases in Arabidopsis. Glycobiology 13, 139-145 (2003).

16. Qin, G. et al. The pomegranate (Punica granatum L.) genome and the genomics of punicalagin biosynthesis. Plant J. 91, 1108-1128 (2017).

17. Yuan, Z. et al. The pomegranate (Punica granatum L.) genome provides insights into fruit quality and ovule developmental biology. Plant Biotechnol. J. 16, 1363-1374 (2018).

18. Park, J., Bae, S. \& Kim, J.-S. Cas-Designer: a web-based tool for choice of CRISPRCas9 target sites. Bioinformatics 31, 4014-4016 (2015).

19. Zuker, M. Mfold web server for nucleic acid folding and hybridization prediction. Nucleic Acids Res. 31, 3406-3415 (2003).

20. Mao, Y. et al. Application of the CRISPR-Cas system for efficient genome engineering in plants. Mol. Plant 6, 2008-2011 (2013).

21. Clark, J. Cetyltrimethyl ammonium bromide (CTAB) DNA miniprep for plant DNA isolation. Cold Spring Harb. Protoc. 2009, pdb.prot5177 (2009).

22. Wilson, A. E., Wu, S. \& Tian, L. PgUGT95B2 preferentially metabolizes flavones/ flavonols and has evolved independently from flavone/flavonol UGTs identified in Arabidopsis thaliana. Phytochemistry 157, 184-193 (2019).
23. Kim, D., Langmead, B. \& Salzberg, S. L. HISAT: a fast spliced aligner with low memory requirements. Nat. Methods 12, 357-360 (2015).

24. Li, B. \& Dewey, C. N. RSEM: accurate transcript quantification from RNA-Seq data with or without a reference genome. BMC Bioinformatics 12, 323 (2011).

25. Love, M. I., Huber, W. \& Anders, S. Moderated estimation of fold change and dispersion for RNA-seq data with DESeq2. Genome Biol. 15, 550-550 (2014).

26. Bradford, M. A rapid and sensitive for the quantitation of microgram quantities of protein utilizing the principle of protein-dye binding. Anal. Biochem. 72, 248-254 (1976).

27. Wilson, A. E. et al. Characterization of a UGT84 family glycosyltransferase provides new insights into substrate binding and reactivity of galloylglucose ester-forming UGTs. Biochemistry 56, 6389-6400 (2017).

28. Edgar, R. MUSCLE: multiple sequence alignment with high accuracy and high throughput. Nucleic Acids Res. 32, 1792-1797 (2004).

29. Kumar, S., Stecher, G. \& Tamura, K. MEGA7: molecular evolutionary genetics analysis version 7.0 for bigger datasets. Mol. Biol. Evol. 33, 1870-1874 (2016). 